\title{
APPLICATION OF HALANAY INEQUALITY TO THE ESTABLISHMENT OF THE EXPONENTIAL STABILITY OF DELAYED COMPARTMENTAL SYSTEM
}

\begin{abstract}
SARIYASA
Abstract. The dynamical convergence of a compartmental system with transport delays is studied. An easily verifiable delay independent sufficient condition for the system to be globally exponentially stable is obtained. Halanay differential inequality is employed to establish the global exponential stability.
\end{abstract}

\section{INTRODUCTION}

Compartmental systems are widely used as mathematical models to describe exchange of material among compartments in biology, medicine, and ecology. A compartment is characterized by the type and amount of material it contains and it is a hypothetical container or pool. Mathematical models are formulated in the form of differential equations with the assumption that the material flows among compartment instantly. This is not true in general since it takes time to transport material from one compartment to another. This situation is easily visualized as one where compartments are connected by (imaginary) pipes so that the material requires time to flow through the pipes. Such systems are called compartmental models with pipes [3].

In several physiological systems involving the transport of tracers of blood, for instance, there is usually a finite time needed for the material to flow from one compartment to another (e.g. from right ventricle to left ventricle). Thus it is worthwhile and necessary to incorporate this transport time (time delay) in the

Received 24 March 2006, revised 23 October 2006, accepted 15 November 2006.

2000 Mathematics Subject Classification: 34K20, 92B05.

Key words and Phrases: Halanay inequality, exponential stability, compartmental system, delay. 
mathematical models of the compartment systems. These models serve as a more realistic models for the compartmental systems

In this paper we investigate the exponential stability of equilibrium of an compartmental model with time delays. The result on exponential stability is of great significance. In addition to the fact that global exponential stability of a system implies global stability of the system with a bigger rate of convergence to equilibrium. Thus, a global exponentially stable system has better performance. We employ Halanay inequality to derive delay independent sufficient conditions. The absence of delay in the condition will greatly increase the convergence time.

\section{EXPONENTIAL STABILITY OF DELAYED COMPARTMENTAL SYSTEM}

We consider a continuous-time compartmental system consisting of $m$ compartments with "transport delays" described by the system of delay differential equations of the form

$$
\frac{d x_{i}(t)}{d t}=-a_{i i} x_{i}(t)+\sum_{\substack{j=1 \\ j \neq i}}^{m} a_{i j} x_{j}\left(t-\tau_{i j}\right)+u_{i}, \quad i=1,2,3, \ldots, m, \quad t>0
$$

This system is an adaptation of one found in [2]. In the subsequent discussion the set $\{1,2,3, \ldots, m\}$ will be denoted by $\mathcal{I}$. In $(1), \tau_{i j} \geq 0, i, j \in \mathcal{I}, i \neq j$ are transport delays; $x_{i}(t)$ denotes the amount of material in the $i$ th compartment at $t$; the nonhomogeneous term $u_{i} \geq 0$ denotes a constant rate of input of the material into the system; $a_{i j} x_{j}(t)$ can be interpreted as the rate at which material reaches the $i$ th compartment from the $j$ th compartment. The system (1) is supplemented with the initial condition of the form

$$
x_{i}(s)=\varphi_{i}(s), \quad s \in[-\tau, 0], \quad i \in \mathcal{I}
$$

in which $\tau=\max _{i, j \in \mathcal{I}}\left\{\tau_{i j}\right\}$.

We denote the equilibrium of (1) by a vector $\mathbf{x}^{\star}=\left(x_{1}^{\star}, x_{2}^{\star}, \ldots, x_{m}^{\star}\right)^{T}$ where

$$
a_{i i} x_{i}^{\star}=\sum_{\substack{j=1 \\ j \neq i}}^{m} a_{i j} x_{j}^{\star}+u_{i}, \quad i \in \mathcal{I}
$$

We first establish the existence and uniqueness of the equilibrium $\mathbf{x}^{\star}$ of (1).

Theorem 2.1. Suppose the constant parameters of (1) satisfy the following:

$$
\begin{aligned}
& a_{i j} \geq 0, \quad i, j \in \mathcal{I}, \quad i \neq j \\
& a_{i i}-\sum_{\substack{j=1 \\
j \neq i}}^{m} a_{j i}>0, \quad i \in \mathcal{I} .
\end{aligned}
$$


Then the system (1) has a unique equilibrium point.

Proof. Consider the following linear system of equations in the unknowns $x_{j}^{\star}, j \in \mathcal{I}$ :

$$
a_{i i} x_{i}^{\star}-\sum_{\substack{j=1 \\ j \neq i}}^{m} a_{i j} x_{j}^{\star}=u_{i}, \quad i \in \mathcal{I} .
$$

It follows from (3) and (4) that $a_{i i}>0$ and that matrix $B=\left(b_{i j}\right)$, with $b_{i i}=a_{i i}>0$, $i \in \mathcal{I}$ and $b_{i j}=-a_{i j} \leq 0, i, j \in \mathcal{I}, i \neq j$, is column diagonally dominant. The matrix $B$ is an $M$-matrix such that $B$ is nonsingular and the elements of $B^{-1}$ are nonnegative. Hence the system (5) has a solution

$$
\mathbf{x}^{\star}=B^{-1} \mathbf{u} \geq 0,
$$

that is an equilibrium of (1).

We proceed to show the uniqueness of the equilibrium $\mathbf{x}^{\star}$. Suppose $\mathbf{x}^{\star}=$ $\left(x_{1}^{\star}, x_{2}^{\star}, \ldots, x_{m}^{\star}\right)^{T}$ and $\mathbf{y}^{\star}=\left(y_{1}^{\star}, y_{2}^{\star}, \ldots, y_{m}^{\star}\right)^{T}$ are two equilibria. We then have the following

$$
a_{i i}\left(x_{i}^{\star}-y_{i}^{\star}\right)=\sum_{\substack{j=1 \\ j \neq i}}^{m} a_{i j}\left(x_{j}^{\star}-y_{j}^{\star}\right), \quad i \in \mathcal{I}
$$

which leads to

$$
a_{i i}\left|x_{i}^{\star}-y_{i}^{\star}\right| \leq \sum_{\substack{j=1 \\ j \neq i}}^{m} a_{i j}\left|x_{j}^{\star}-y_{j}^{\star}\right|, \quad i \in \mathcal{I}
$$

and hence

$$
\sum_{i=1}^{m}\left\{a_{i i}-\sum_{\substack{j=1 \\ j \neq i}}^{m} a_{j i}\right\}\left|x_{i}^{\star}-y_{i}^{\star}\right| \leq 0 .
$$

By applying the condition (4) on (6) we conclude that $x_{i}^{\star}=y_{i}^{\star}$ for all $i \in \mathcal{I}$. The uniqueness of the equilibrium $\mathbf{x}^{\star}$ of (1) follows. We have shown the existence and uniqueness of the equilibrium $\mathbf{x}^{\star}$ of (1). The proof is complete.

In the following we show that the same conditions as in (3) and (4) guarantee the global exponential stability of the equilibrium point $\mathbf{x}^{\star}$. We use Halanay inequality $[4$, p. 378]) to establish this result. Halanay inequality in comparison to Lyapunov functional is rarely used in the study of stability despite its possible generalizations and applications $[1,2,5]$. The proof of the following theorem is based on the results found in [6]. First we state a scalar version of Halanay inequality $([2])$ as follows.

Halanay inequality. Let $\alpha, \beta$ and $\tau$ be positive numbers and let $u(t)$ be a nonnegative solution of

$$
\frac{d u(t)}{d t} \leq-\alpha u(t)+\beta\left(\sup _{s \in[t-\tau, t]} u(s)\right), \quad t>0 .
$$


If $\alpha>\beta$, then there exist constants $\sigma>0$ and $k>0$ such that $u(t) \leq k e^{-\sigma\left(t-t_{0}\right)}$ for $t>t_{0}, t_{0} \in \mathbb{R}$.

Theorem 2.2. Suppose (3) and (4) hold. Then the system (1) is globally exponentially stable in the sense that there exists a constant $\mu>0$ such that

$$
\left|x_{i}(t)-x_{i}^{\star}\right| \leq e^{-\mu t} \max _{i \in \mathcal{I}}\left(\sup _{s \in[-\tau, 0]}\left|x_{i}(s)-x_{i}^{\star}\right|\right), \quad i \in \mathcal{I}, \quad t>0, \quad \tau=\max _{i \in \mathcal{I}}\left\{\tau_{i j}\right\}
$$

where $x_{i}^{\star}$ is the equilibrium point of (1)

Proof. We define $G_{i}:[0, \infty) \longmapsto \mathbb{R}, i \in \mathcal{I}$ as follows:

$$
G_{i}\left(\mu_{i}\right)=a_{i i}-\mu_{i}-\sum_{\substack{j=1 \\ j \neq i}}^{m} a_{j i} e^{\mu_{i} \tau_{j i}}, \quad \mu_{i} \in[0, \infty), \quad i \in \mathcal{I}
$$

We note that $G_{i}(0)>0$ owing to (4). By the continuity of $G_{i}$ on $[0, \infty)$ and noting that $G_{i}\left(\mu_{i}\right)$ is decreasing, there exists $\mu_{i}^{\star} \in(0, \infty)$ such that $G_{i}\left(\mu_{i}^{\star}\right)=0$. Let $\mu=\min _{i \in \mathcal{I}}\left\{\mu_{i}^{\star}\right\}$ and consequently we have

$$
G_{i}(\mu)=a_{i i}-\mu-\sum_{\substack{j=1 \\ j \neq i}}^{m} a_{j i} e^{\mu \tau_{j i}} \geq 0, \quad i \in \mathcal{I}
$$

From (1) and (2) we derive that

$$
\frac{d}{d t}\left(x_{i}(t)-x_{i}^{\star}\right)=-a_{i i}\left(x_{i}(t)-x_{i}^{\star}\right)+\sum_{\substack{j=1 \\ j \neq i}}^{m} a_{i j}\left(x_{j}\left(t-\tau_{i j}\right)-x_{j}^{\star}\right), \quad i \in \mathcal{I}, \quad t>0 .
$$

Now, evaluating upper right derivative of (8) yields

$$
\frac{d^{+}}{d t}\left|x_{i}(t)-x_{i}^{\star}\right| \leq-a_{i i}\left|x_{i}(t)-x_{i}^{\star}\right|+\sum_{\substack{j=1 \\ j \neq i}}^{m} a_{i j}\left|x_{j}\left(t-\tau_{i j}\right)-x_{j}^{\star}\right|, \quad i \in \mathcal{I}, \quad t>0 .
$$

We define $z_{i}(t)$ as follows

$$
z_{i}(t)=e^{\mu t}\left|x_{i}(t)-x_{i}^{\star}\right|, \quad t \geq-\tau, \quad i \in \mathcal{I} .
$$

Using (9) and (10) we derive that

$$
\begin{aligned}
\frac{d^{+} z_{i}(t)}{d t} & \leq-\left(a_{i i}-\mu\right) z_{i}(t)+\sum_{\substack{j=1 \\
j \neq i}}^{m} a_{i j} e^{\mu \tau_{i j}} z_{j}\left(t-\tau_{i j}\right), \quad i \in \mathcal{I}, \quad t>0 \\
& \leq-\left(a_{i i}-\mu\right) z_{i}(t)+\sum_{\substack{j=1 \\
j \neq i}}^{m} a_{i j} e^{\mu \tau_{i j}}\left(\sup _{s \in[-\tau, 0]} z_{j}(s)\right)
\end{aligned}
$$


where $\tau=\max _{i, j \in \mathcal{I}}\left\{\tau_{i j}\right\}$. Let

$$
M=\max _{i \in \mathcal{I}}\left\{\sup _{s \in[-\tau, 0]}\left|x_{i}(s)-x_{i}^{\star}\right|\right\}>0 .
$$

It follows from (10) and (12) that $z_{i}(t) \leq M$ for all $i \in \mathcal{I}, t \in[-\tau, 0]$. We claim that

$$
z_{i}(t) \leq M \quad \text { for all } \quad i \in \mathcal{I}, \quad t>0 .
$$

We now prove the claim in (13) as follows. Suppose that (13) is not valid; then there exists an $i=p \in \mathcal{I}$ and a $t_{1}>0$ such that

$$
\left.\begin{array}{ll}
z_{i}(t) \leq M & \text { for } \quad i \in \mathcal{I}, \quad i \neq p, \quad t \in\left[-\tau, t_{1}\right] \\
z_{p}(t) \leq M & \text { for } \quad t \in\left[-\tau, t_{1}\right], \quad z_{p}\left(t_{1}\right)=M, \quad \frac{d^{+} z_{p}\left(t_{1}\right)}{d t}>0 .
\end{array}\right\}
$$

But we have from (11) and (14),

$$
\begin{aligned}
0<\frac{d^{+} z_{p}\left(t_{1}\right)}{d t} & \leq-\left(a_{p p}-\mu\right) z_{p}\left(t_{1}\right)+\sum_{\substack{j=1 \\
j \neq p}}^{m} a_{p j} e^{\mu \tau_{p j}}\left(\sup _{s \in\left[t_{1}-\tau, t_{1}\right]} z_{j}(s)\right) \\
& \leq-\left(a_{p p}-\mu-\sum_{\substack{j=1 \\
j \neq p}}^{m} a_{p j} e^{\mu \tau_{p j}}\right) M \leq 0, \quad \text { by }(7)
\end{aligned}
$$

which is not possible. Hence (13) holds. We then use (10) in (13) to obtain

$$
\left|x_{i}(t)-x_{i}^{\star}\right| \leq e^{-\mu t} \max _{i \in \mathcal{I}}\left\{\sup _{s \in[-\tau, 0]}\left|x_{i}(s)-x_{i}^{\star}\right|\right\} \quad \text { for all } \quad i \in \mathcal{I}, \quad t>0 .
$$

Since $\mu>0$, the exponential convergence of all solutions of (1) towards $\mathbf{x}^{\star}$ follows. This completes the proof.

\section{CONCLUDING REMARKS}

We have studied the dynamical convergence of a compartmental system with delays. A sufficient condition for the global exponential stability of the delayed compartmental system is derived. This condition is formulated in terms of the parameters of the system; such a condition is easy to verify. We have demonstrated the use of Halanay inequality in establishing the global exponential stability of the system.

Acknowledgement. The author wishes to thank the referee(s) for the helpful suggestions. 


\section{REFERENCES}

1. C.T.H. BAKER AND A. TANG, Generalised Halanay inequalities for Volterra functional differential equations and discretised versions, Report no. 299, Manchester Centre for Computational Mathematics, The University of Manchester, England, 1996.

2. K. Gopalsamy, Stability and oscillations in delay differential equations of population dynamics, Kluwer Academic Publishers, The Netherland, 1992.

3. I. Györi AND J. Eller, "Compartmental systems with pipes", Math. Biosci. 53 (1981), 223-247.

4. A. Halanay, Differential equations: stability, oscillation, time lags, Academic Press, New York, 1966.

5. S. Mohamad and K. Gopalsamy, "Continuous and discrete Halanay-type inequalities", Bull. Aust. Math. Soc. 61 (2000), 371-385.

6. S. Mohamad, AND K. Gopalsamy, "Exponential stability of continuous-time and discrete-time cellular neural networks with delays", Appl. Math. Comput. 135 (2003), $17-38$.

SARIYAsa: Jurusan Pendidikan Matematika, Universitas Pendidikan Ganesha, Singaraja 81116, Indonesia.

E-mail: sariyasa64@yahoo.com 